\title{
Statyba
}

\section{THE INDUCED OSCILLATIONS OF FLEXIBLE PRESTRESSED ELEMENTS OF STRUCTURES (SYMMETRICAL SYSTEM)}

\section{I. Kazakevič \& V. E. Volkova}

To cite this article: M. I. Kazakevič \& V. E. Volkova (2000) THE INDUCED OSCILLATIONS OF FLEXIBLE PRESTRESSED ELEMENTS OF STRUCTURES (SYMMETRICAL SYSTEM), Statyba, 6:1, 55-59, DOI: $10.1080 / 13921525.2000 .10531564$

To link to this article: https://doi.org/10.1080/13921525.2000.10531564

Published online: 26 Jul 2012.

Submit your article to this journal $₫$

Џ Article views: 54 


\title{
ВЫНУЖДЕННЫЕ КОЛЕБАНИЯ ГИБКИХ ПРЕДНАПРЯЖЕННЫХ ЭЛЕМЕНТОВ КОНСТРУКЦИЙ
}

\author{
М. И. Казакевич, В. Е. Волкова
}

\section{1. Предисловие}

Представлены результаты аналитического и численного исследований вынужденных колебаний предварительно напряженньх гибких элементов конструкцшй. Рассмотрены два случая центрального предварительного напряжения: без промежуточного крепления затяжки к гибкому элементу и с промежуточным креплением в середине длины элемента. Рассматриваемая физическая модель, помимо гибких элементов, применима к гибким пологим оболочкам и аркам, мембранам, болыпим космическим антенным полям (БКАП).

Исследуемые системы при определенных соотношениях физических параметров имеют несколько несмежных форм равновесия. Переход от одной устойчивой формы равновесия к другой, несмежной, форме происходит скачкообразно. В этом случае они называются системами с "перескоком", или системами с двумя потенциальными “ямами". Отличительной особенностью данного класса систем является возможность сушествования несмежных устойчивых режимов колебаний при фиксированной частоте возмущения. Возможность реализации того или иного устойчивого режима колебаний сложным образом зависит от начальных условий [1]. Численное моделирование вынужденных колебаний систем с "перескоком" подробно обсуждалось в работе [2].

\section{2. Анализ вынужденных колебаний системы}

Вынужденные колебания систем с "перескоком" описываются нелинейным дифференциальным уравнением типа Дуффинга:

$$
\ddot{\phi}+\varepsilon \dot{\phi}-\alpha \phi+\beta \phi^{3}=P_{1} \cos (\omega t+\theta),
$$

где $\phi$ - обобщенная координата поперечных перемешений средней по длине стержня точки; $\varepsilon$ - коэффициент демпфирования; $\alpha, \beta-$ коэффициенты, опре- деляющие характер восстанавливаюшей силы $R(\phi)=-\alpha \phi+\beta \phi^{3} ; P_{1}, \omega$ - параметры внешнего возмущения.

Отметим, что при свободных колебаниях ( $P_{1}=0$ ) [1] система (1) имеет три положения равновесия, два из которых $\phi_{b, c}= \pm \sqrt{\alpha / \beta}-$ устойчивы, а третье - промежуточное $\phi_{a}=0-$ неустойчиво.

Известно, что в зависимости от значения потенциальной энергии возможно существование одного из трех устойчивых режимов колебаний (рис. 1): а) “большие" колебания относительно всех трех положений равновесия (точки $\boldsymbol{a}, \boldsymbol{b}$ и $\boldsymbol{c}$ ); б) “малые” колебания относительно левого положения равновесия (точка b); в) "малье" колебания относительно правого положения равновесия (точка $c$ ). При этом “большие" колебания обладают свойствами жесткой системы, а "малые" колебания - свойствами мягкой системы.

\section{3. Аналитическое исследование вынужденных колебаний}

\section{1. "Большие" колебания}

Ранее отмечалось, что “большие" колебания в пределах пирокого диапазона частот возмущения $\omega$ имеют явно выраженный установившийся характер моногармонического процесса на основной частоте. Это позволяет утверждать, что приближенное решение может дать достоверную оценку областей параметров системы, в которых имеют место “большие" колебания.

Решение уравнения (1) будем искать в виде:

$$
\phi=A \cos \omega t \text {. }
$$

Для получения приближенного решения используем метод гармонического баланса. Подставляя выражение (2) в уравнение (1) и применяя известные тригонометрические преобразования, получим следующее 


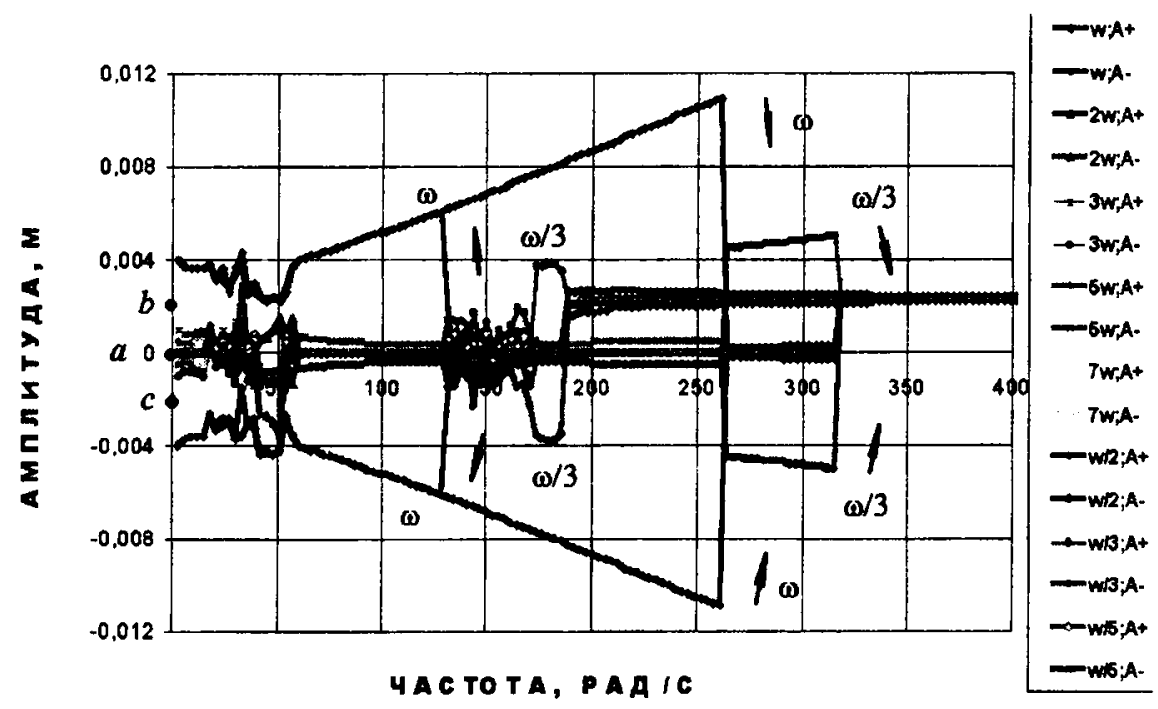

Рис. 1. Влияние ультра- и субгармонических составляюших на характер колебаний в симметричных системах с "перескоком". $\alpha=40,81 / \mathrm{c}^{2} ; \beta=76600001 / \mathrm{s}^{2} \mathrm{c}^{2} ; \varepsilon=0,51 / \mathrm{c} ; \quad P_{1}=0,15 \mathrm{~m} / \mathrm{c}^{2}$

Fig 1. Influence of ultra and subharmonic elements on the character of oscillations in symmetrical systems

выражение для определения собственной частоты “больших" колебаний:

$$
\omega_{*}^{2}(A)=-\alpha+\frac{3}{4} \beta A^{2} \quad \text { npu } \quad A^{2}>\frac{4 \alpha}{3 \beta} .
$$

Таким образом, собственная частота линеаризованной системы является действительной лишь при достаточно больших значениях амплитуды.

Значение фазового угла и частоты вынужденных колебаний системы определяются зависимостями:

$$
\begin{gathered}
\operatorname{tg} \theta=\frac{\varepsilon \omega}{\omega_{*}^{2}(A)-\omega^{2}}=\frac{\varepsilon \omega}{-\alpha+\frac{3}{4} \beta A^{2}-\omega^{2}}, \\
\omega^{2}=\omega_{*}^{2}(A)-\frac{\varepsilon^{2}}{2} \pm \sqrt{\frac{\varepsilon^{4}}{4}-\varepsilon^{2} \omega_{*}^{2}(A)+\left(\frac{P_{1}}{A}\right)^{2}} .
\end{gathered}
$$

В случае малого демпфирования уравнение (5) может быть приведено к виду:

$$
\omega^{2}=\omega_{*}^{2}(A) \pm \sqrt{\left(\frac{P_{1}}{A}\right)^{2}-\varepsilon^{2} \omega_{*}^{2}}
$$

Дифференцируя по частоте и амплитуде уравнение (5) и приравнивая полученные производные к нулю, получим уравнения кривых, ограничивающих области неустойчивых “больших" колебаний основного тона:

$$
\omega_{1}=\sqrt{-\alpha+\frac{3}{4} \beta A^{2}-\frac{\varepsilon^{2}}{2}},
$$

$$
\omega_{2}=\sqrt{-\alpha+\frac{3}{4} \beta A_{s}^{2}+\frac{2 P_{1}^{2}}{3 \beta A_{s}{ }^{2}}} .
$$

\section{2. "Малые" колебания}

Вынужденные колебания относительно точек $\boldsymbol{b}$ и c описываются дифференциальным уравнением вида:

$$
\bar{\phi}+\varepsilon \bar{\phi}+2 \alpha \bar{\phi} \pm 3 \sqrt{-\alpha \beta} \bar{\phi}^{2}+\beta \bar{\phi}^{3}=P_{1}(\cos \omega t+\theta),
$$

где $\phi=\bar{\phi} \pm \sqrt{\alpha / \beta}$.

Известно, что "малые" колебания в резонансных диапазонах частот $\omega$ имеют явно выраженный установившийся характер моногармонического процесса на основной частоте.

Решение уравнения (8) будем искать в виде:

$$
\bar{\phi}=A_{s} \cos \omega_{s} t
$$

Применив метод гармонического баланса и выполнив преобразования, аналогичные приведенным выше, получим выражения для фазового угла и частоты вынужденных колебаний:

$$
\begin{gathered}
\operatorname{tg} \theta=\frac{\varepsilon \omega_{s}}{\omega_{*}^{2}\left(A_{s}\right)-\omega_{s}^{2}}=\frac{\varepsilon \omega_{s}}{2 \alpha+\frac{3}{4} \beta A_{s}^{2}-\omega_{s}^{2}}, \\
\omega_{s}^{2}=2 \alpha+\frac{3}{4} \beta A_{s}^{2}-\frac{\varepsilon^{2}}{2} \pm \\
\pm \sqrt{\frac{\varepsilon^{2}}{4}-\varepsilon^{2}\left(2 \alpha+\frac{3}{4} \beta A_{s}^{2}\right)+\left(\frac{P_{1}}{A_{s}}\right)^{2}},
\end{gathered}
$$


где собственная частота "малых" колебаний для симметричных систем определяется выражением:

$$
\omega_{*}^{2}(A)=2 \alpha+\frac{3}{4} \beta A_{s}^{2} .
$$

В случае малого демпфирования выражение (13) может быть преобразовано к виду:

$$
\omega_{s}^{2}=2 \alpha+\frac{3}{4} \beta A_{s}^{2}-\frac{\varepsilon^{2}}{2} \pm \sqrt{\left(\frac{P_{1}}{A_{s}}\right)^{2}-\varepsilon^{2}\left(2 \alpha+\frac{3}{4} \beta A_{s}^{2}\right)}
$$

Области неустойчивых “малых" колебаний ограничены кривыми, описываемыми следующими уравнениями:

$$
\begin{gathered}
\omega_{1 s}=\sqrt{2 \alpha+\frac{3}{4} \beta A_{s}^{2}-\frac{\varepsilon^{2}}{2}}, \\
\dot{\omega}_{2 s}=\sqrt{2 \alpha+\frac{3}{4} \beta A_{s}^{2}+\frac{2 P_{1}^{2}}{3 \beta A_{s}^{4}}} .
\end{gathered}
$$

\section{4. Алгоритм и методика численного эксперимента}

Для получения на ЭВМ численного решения уравнения (1) была создана программа. В основу процедуры численного моделирования был предложен метод Рунге-Кутта 4-го порядка. Учитывая сложный характер колебаний нелинейных систем, опнсываемых уравнением (1), шаг интегрирования $\Delta t$ выбирался в диапазоне $T / 250 \leq \Delta t \leq T / 150$, где $T=2 \pi / \omega$ - период гармонической составляошей внешнего воздействия. Выполнение этого условия обеспечивало устойчивость процедуры численного решения уравнения (1) при всех рассмотренных вариантах значений его коэффициентов. Учитывая возможность возникновения многотоновых колебаний, был предусмотрен блок спектрального анализа. Для определения амплитуд этих гармонических составляющих был использован алгоритм Герцеля.

\section{5. Результаты аналитического и численного моделирования вынужденных колебаний при гармоническом нагружении}

Исследуем вынужденные колебания модели элемента больших космических антенн. Положим, что расчетная схема исследуемой системы может быть представлена в виде стального однородного стержня длиной $l=3 \mu$, с поперечным сечением $b \times h=0,3 \times$ $\times 0,004 \mu, c$ затяжкой, которая крепится к центрам крайних поперечных сечений. Натяжение затяжки
$N^{*}=720 H$. Опирание стержня шарнирное. В соответствии с [3] коэффициенты $\alpha$ и $\beta$ принимают следующие знацения $\alpha=40,81 / c^{2}, \beta=76600001 /(\text { мс })^{2}$.

Исследуемая система имеет три положения равновесия, два из которых устойчивые $y_{b, c}= \pm 0,0023 \mathrm{~s}$ и третье - среднее - неустойчивое $y_{a}=0$. Бифуркационные точки разделяют зоны существования “больших" и "мальх" колебаний [3].

Параметр демпфирования $\varepsilon$ и амплитуда гармонического нагружения $P_{1}$ в вычислительном зксперименте принимали следующие значения: $\varepsilon=0,1 ; 0,5$; $1,0 c^{-1} ; P_{1}=0,15 ; 0,3 ; 1,5 \mathrm{mc}^{-2}$. Частота $\omega$ изменялась в пределах $\omega_{\text {на }+} \leq \omega \leq \omega_{\text {кон }}$ с шагом $\Delta \omega=1$ pad $/ c$.

Анализ результатов численного интегрирования показал, что при увеличении частоты система вначале совершает "большие" колебания вплоть до так называемой “частоты срыва" $\omega_{\phi}$, после которой устанавливается режим "малых" колебаний. Значение частоты $\omega_{\phi p}$ естественно зависит от коэффипиента демпфирования и уменьшается с его ростом. При уменњшения частоты реализуются "малые" колебания вокруг одного из устойчивых положений равновесия, а затем при некотором значения частоты $\omega_{y c m}<\omega_{c p}$ устанавливается режим "больших" колебаний. С увеличением значения параметра демпфирования $\varepsilon$ диапазон $\left(\omega_{c p}-\omega_{y c m}\right)$ многозначности амплитуд основного тона установившихся колебаний систем с прощелкиванием уменьшается, как и в случае жестких нелинейных систем Дуффинга.

Следует отметить, что при "прямом" проходе, когда $\omega$ равно или несколько больше $\omega_{p p}$, доминируют субгармонические колебания с частотой $\omega / 3$, амплитуды которых сушественно больше амплитуд основного тона (см. рис. 1), т.е. переход от "больших" колебанй основного тона к "малым" происходит не мгновенно, а через субгармонические тона с частотой $\omega / 3$.

Аналогичная картина наблюдается при “обратном" проходе с той лишь разницей, что доминирующие субгармонические колебания возникают с частотой w/2 и это явление наступает незадолго до момента установления режима "больших" колебаний основного тона.

Результаты моделирования показали, что в некоторых диапазонах частот именно начальные условия определяют реализацию того или иного устойчивого режима колебаний. Для прогнозирования динами- 
a)

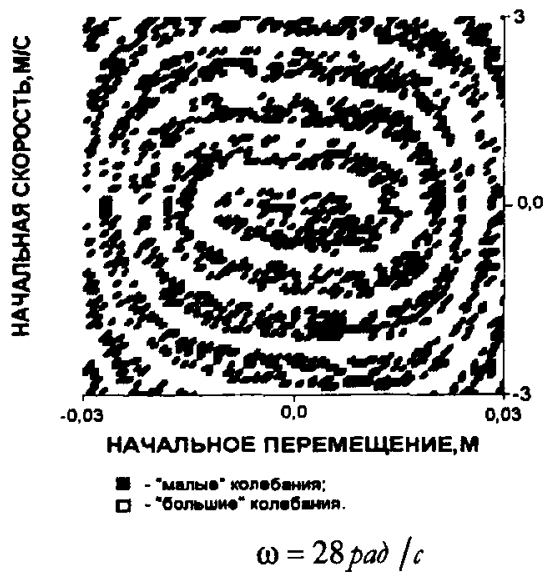

b)

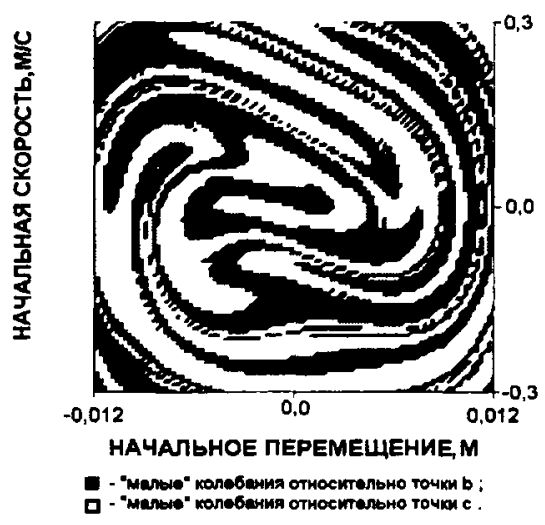

$\omega=40$ pad $/ c$

Рис. 2. Области притяжения устойчивых режимов колебаний. $\alpha=40,8 c^{-2} ; \beta=7660000 M^{2} c^{2} ; \varepsilon=4,0 c^{-1} ; P_{1}=1,5 \mathcal{M c} c^{-2}$

Fig 2. Regions of attraction of stable regimes of oscillations

ческого поведения преднапряженных стержней было разработано программное обеспечение для построения зон притяжения установившихся режимов колебаний. Данная программа позволяет анализировать, какой из трех режимов колебаний реализуется при заданнцхх начальных условиях: а) "болышие" колебания; б) "малые" колебания относительно точки $\boldsymbol{b}$; в) "малые" колебания относительно точки $\boldsymbol{c}$.

На рис. 2 представлены полученњые области притяжения начальных условий установившихся режимов для колебаний в резонансной и зарезонансной зонах. Графики имеют сложную конфигурацию в виде раскручивающейся спирали. При этом чередовакие зон по мере удаления от центра увеличивается, а сами они сужаются. Следует отметить, что границы зон негладкие (фрактальные).

На рис. 3 приведено сопоставление аналитического исследования и численного моделирования, а также "скелетные" кривые для модели преднапряженного элемента. Анализируя рис. 1, можно утверждать, что для широкого диапазона частот аппроксимашия решения уравнения (1) гармоникой основного тона дает удовлетворительную информацию о поведении системы.

\section{6. Выводы}

Получены аналитические и численные решения цифференциального уравнения (1) вынужденных колебаний предварительно напряженных гибких элементов конструкций для случаев «малых" и «больших» колебаний.

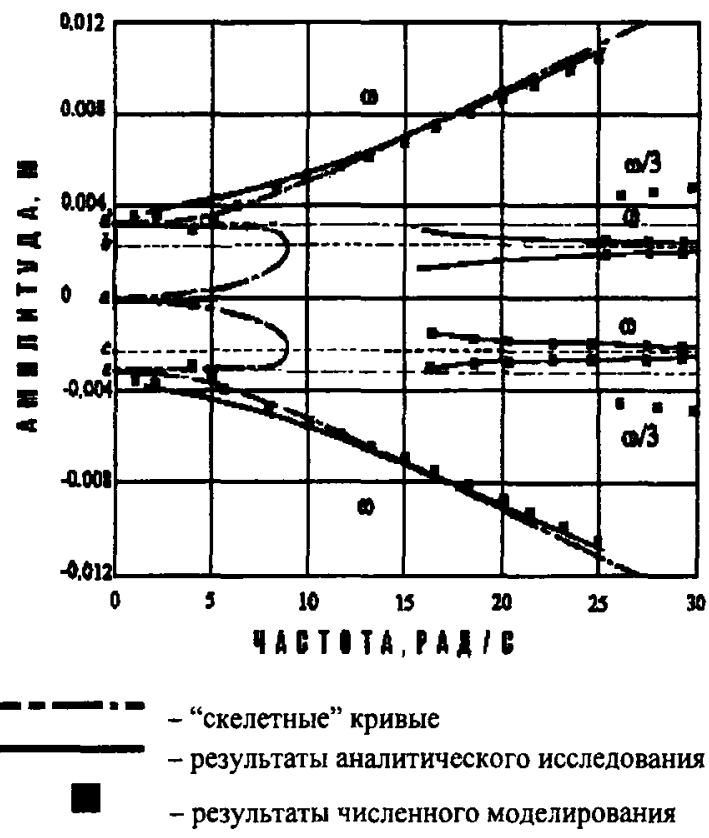

Рис. 3. Амплитудно-частотные характеристики системы (1). $\alpha=40,8 c^{-2}, \beta=7660000(\text { Mc })^{-2}, \varepsilon=0,5 c^{-1}$,

$P_{1}=0,15 \mathcal{M C}^{-2}$

Fig 3. Amplitude-frequency characteristics of the system (1)

ІІри увеличении частоты исследуемая система вначале совершает «большие» колебания вплоть до так называемо частоты срыва $\omega_{c p}$, после которой устанавливается режим «малых» колебаний. Значение частоты $\omega_{c p}$ зависит от коэффициента демпфирования. При уменьшении частоты реализуются малые колебания вокруг одного из устойчивых положений равновесия, а затем уже устанавливается режим «больших» колебаний. 


\section{Литература}

1. М. И. Казакевич, Э. Н. Кваша, С. Ф. Редько. Влияние начальньх условий на характер установившихся колебаний систем с нелинейной восстанавливающей силой // Математическая физика. Вып. 15. Киев: Наукова думка, 1974, с. 59-62.

2. М. И. Казакевич, С. Ф. Редько, В. Е. Волкова. Вынужденные колебания преднапряженных стержней // Тр. междунар. конф. "Теория и практика металлических конструкций”. Том 1. Донецк: Изд-во Донецкой государственной строительной академии, 1997, с. 15-20.

3. М.И. Казакевич, В.Е. Волкова. Точное решение свободных колебаний преднапряженных стержней // Proc. of 5-th International Conference "Modern Building Materials, Structures and Techniques", Vol 3. Vilnius: Technika, 1997, p. 145-150.

Iteikta 19990808

\section{IŠ ANKSTO ITEMPTU LIAUNU KONSTRUKCIJOS ELEMENTU PRIVERSTINIAI SVYRAVIMAI}

\section{I. Kazakevix, V. E. Volkova}

San trauk a

Pateikti is anksto jtemptỵ liauny konstrukcijos elementy dinamiško kitimo tyrimo rezultatai. Tyrinèjamas fizikinis modelis gali büti taikomas ir tokioms liaunoms konstrukcijoms, kaip nuožulnios arkos, kevalai, ilinkusios plokštelès, didelių kosminiu antenų sistemų elementai.

Tokiu sistemy dinaminis kitimas aprašomas lygtimi:

$$
\ddot{\phi}+\varepsilon \dot{\phi}-\alpha \phi+\beta \phi^{3}=P_{1} \cos (\omega t+\theta) \text {. }
$$

$\varepsilon$ - slopimo koeficientas; $\alpha, \beta$ - koeficientas, nusakantis nelinijinc̀s atkuriančios jègos pobūdi $R(\phi)=-\alpha \phi+\beta \phi^{3} ; P_{1}, \omega$ - išorinio poveikio parametrai.

Pagrindinių kreivių analizè rodo, kad sistemos savybès dvireikšmès. Esant ,dideliems" virpesiams pasireiskia standžios sistemos savybès, o esant "mažiems" - minkక̌tos (plastiškos). Kaip parodyta 1 pav., padidinus ar sumažinus trikdžiu dažni, svyravimų amplitudès pasikeitimo pobūdis skiriasi. Priverstinius svyravimus nuo vienos stabilios Łakos i kita lydi ne tik perèjimas nuo "didelių“ svyravimu prie "mažų“ ir atvirkšciai, bet ir kombinaciniu tonų $(2 \omega, 3 \omega 5 \omega, \ldots, \omega / 2, \omega / 3)$ svyravimu atsiradimas.

Harmoninio balanso metodu gauti analitiniai sprendimai „dideliems" ir "mažiems" priverstiniams svyravimams. Sprendimas buvo randamas is $\phi=A \cos \omega t$ - esant "dideliems" svyravimams, $\bar{\phi}=A_{s} \cos \omega_{s} t$ - esant "mažiems" svyravimams, kur $\phi=\bar{\phi} \pm \sqrt{\alpha / \beta}$. Gautos išraiškos kreivèms, apribojancioms nestabilias dažninès amplitudines charakteristikos sritis, ir „dideliu"“ svyravimų amplitudès kritinès reikšmès.

Pateikti skaiciuojamojo eksperimento metodika ir rezultatai. Uždavinio sprendimui būtinai programinei ịrangai sudaryti buvo taikyti skaitınio integravimo metodai (Runge-Kutta eilès metodas), spektrinès analizès (Gercelio algoritmas), kompiuterinès grafikos ir kt. Skaitinio integravimo rezultatai gerai sutampa su analitinio sprendimo rezultatais tiek pagrindinéms kreivèms, tiek ir dažninèms amplitudinèms priverstiniu svyravimu charakteristikoms.

\section{THE INDUCED OSCILLATIONS OF FLEXIBLE PRESTRESSED ELEMENTS OF STRUCTURES (SYMMETRICAL SYSTEM)}

\section{I. Kazakevitch, V. E. Volkova}

\section{Summary}

The results of the investigations of dynamic behaviour of the flexible prestressed structure elements are presented in the paper. The given physical model can be applied to the flexible structures like sloping arches, shells, bending plates, elements of the large space antenna fields (LSAF). The dynamic behaviour of the investigated systems is described by the equations

$$
\ddot{\phi}+\varepsilon \dot{\phi}-\alpha \phi+\beta \phi^{3}=P_{1} \cos (\omega t+\theta),
$$

where $\varepsilon$ is damping coefficient, $\alpha, \beta$ are coefficients determining the character of non-linear restoring force $R(\phi)=-\alpha \phi+\beta \phi^{3} ; P_{1}, \omega$ are parameters of outer effect.

The analysis of the "skeleton" curves disclosed the double qualities of system (1). Thus, "large" oscillations possess the peculiarities of the rigid system behaviour, and "small" oscillations possess the qualities of soft systems. The character of the oscillation amplitude changing with the increase or decrease of the excitation frequencies is followed in Fig 1. The establishment of the forced oscillation regimes from one branch to another is accompanied not only by the transition from "large" oscillations to "small", or vice versa, but also by the development of the combination tones $(2 \omega, 3 \omega 5 \omega, \ldots, \omega / 2, \omega / 3)$.

The analytical solutions for "large" and "small" forced oscillations are given by harmonic balance method. The sclution was found in the form $\phi=A \cos \omega t$ for "large" oscillation, and $\bar{\phi}=A_{s} \cos \omega_{s} t$ for "small" oscillation, where $\phi=\bar{\phi} \pm \sqrt{\alpha / \beta}$. The for curves disclosed unstable branches of amplitude-frequency curves and critical value amplitude of "large" oscillations were obtained.

The methods and results of the computing experiment are presented in the paper. For working out the software necessary for the given task, the method of numerical integration (RungeKutta method of the fourth order), spectral analysis (Hertzel algorithm), computer graphics, etc were used. The results of the numerical integration are well-coordinated with the analytical solution for the "framework" curves and for the amplitudefrequency curves of forced oscillations.

Michael 1. KAZAKEVITCH. Professor, Doctor of Sciences Dept of Bridge Construction. Dnepropetrovsk State Technical University of Railway Transport, Lazarian Str. 2, 320010 Dnepropetrovsk, Ukraine.

Doctor of Sciences (1982, aerodynamics of bridges). Since 1983 Professor of Dept of Structural Mechanics, Bridge Construction. Author of more than 250 scientific publications, including 10 books and 17 patents. Research interests: dynamics, aerodynamics of bridges, buildings; stabilisation of structures; non-linear oscillations and chaos.

Victoria E. VOLKOVA. Doctor. Dept of Building Structures. Dnepropetrovsk State Technical University of Railway Transport, Lazarian Str. 2, 320010 Dnepropetrovsk, Ukraine.

Dr (1999, structural mechanics). Author of 14 scientific publications. Research interests: dynamics, aerodynamics of bridges, buildings; non-linear oscillations and chaos. 